\title{
On the Pre-metric Formulation and Nonlinearization of Charge-free Electrodynamics
}

\author{
Stoil Donev*, Maria Tashkova, \\ Institute for Nuclear Research and Nuclear Energy, \\ Bulg.Acad.Sci., 1784 Sofia, blvd.Tzarigradsko chaussee 72, Bulgaria
}

\begin{abstract}
This paper presents a coordinate free pre-metric formulation of charge free Maxwell-Minkowski electrodynamics, and of the developed by the authors non-linear Extended Electrodynamics. First we introduce some formal relations from multilinear algebra and differential geometry to be used further. Then we recall and appropriately modify the existing pre-metric formulation of linear charge free electrodynamics in pre-relativistic and relativistic forms as preparation to turn to corresponding pre-metric nonlinearization. After some preliminary examples and notes on nonlinearization, we motivate our view for existence and explicit formulation of time stable subsystems of the physical field objects considered. Section 5 presents the formal results of our approach on the pre-metric nonlinear formulations in static case, in time-dependent case, and in space-time formulation. In the Conclusion we give our general view on "why and how to nonlinearize". The Appendix gives a possible formal extension of our aproach to many subsystem field objects.
\end{abstract}

keywords: extended electrodynamics, electromagnetic field objects, pre-metric, nonlinearization.

\section{Introduction}

We start with some general remarks concerning our view on physical systems and the necessary physical motivations when a theoretical model is meant to be appropriately constructed. Appropriate here means that, the description of structure and behavior must be based on the natural assumption that physical world demonstrates itself through relatively stable objects, being able to survive, or to be destroyed, during mutual interactions. Our knowledge about physical world comes namely from this mutual interaction, no matter weather this interaction is direct, or indirect. We should not equalize the concepts of explanation and description, our view is that theoretical physics must explain any description of natural objects and processes. So, physical motivations should lead to explanation of the description model built. Every built model must give: first, corresponding mathematical structure of the physical system studied, which includes constituents + subsystems, second, mathematical images of the physical characteristics by means of which the system considered demonstrates existence and participation among the other systems. For example, it is one thing to model natural flows by vector fields, and another thing to calculate the exchanged energymomentum between these flows. We may conclude that mathematical models should rather give equations of balance of corresponding quantities, than equations that equalize the mathematical images of the interacting systems. For example, Poynting's equation $\operatorname{div}(\mathbf{E} \times \mathbf{B})=-\frac{1}{2 c} \frac{\partial}{\partial t}\left(\mathbf{E}^{2}+\mathbf{B}^{2}\right)$

*e-mail:sdonev@inrne.bas.bg 
is a balance relation, while Maxwell equation $\frac{1}{c} \frac{\partial \mathbf{E}}{\partial t}=\operatorname{curl} \mathbf{B}$ equalizes differentiated mathematical images.

Any physical system has spatial structure and shows definite stability properties, so, it can support its existence and compensate in definite degree the external disturbances through appropriate shape changes and kinematical behavior without losing identity. Shortly speaking, its time existence is a dynamical process being strongly connected with various and continuous internal and external stress-energy-momentum exchange processes. All these processes are real phenomena and any attempt for their description should be based on appropriate mathematical structures. Of course, all real changes during existence of a physical system also must be formally identified with appropriate mathematical objects. Clearly, if we are going to describe formally a real physical system together with all admissible changes, the corresponding mathematical images of the system, including its recognizable subsystems and all real changes, must have tensor nature in order not be possible to be removed by coordinate transformations.

All these moments suggest to pay due respect to modern differential geometry as appropriate mathematical language. In fact, the more than a century intensive interaction between differential geometry and theoretical and mathematical physics proved to be exclusively useful, suggestive and creative process.

The plan of the paper is the following.

First we introduce some formal relations from multilinear algebra and differential geometry to be used further. Then in sections 2-3 we start the pre-metric approach in the linear charge free electrodynamics in pre-relativistic and relativistic forms as preparation to turn to corresponding pre-metric nonlinearization. Section 4 gives some preliminary examples and notes and on nonlinearization, and motivates our view for existence and explicitly formulation of time stable subsystems of the physical objects considered. Section 5 presents the pre-metric formulation in the static case, in time-dependent case, and in space-time formulation. In the Conclusion we give our general view on "why and how to nonlinearize".

In the next section we are going to introduce the objects and relations to be used further in the paper. Our basic mathematical object will be an oriented manifold $(M, \omega)$ with appropriate dimension. The orientation defined by $\omega$ will allow to compute integral characteristics of the objects considered on one hand, and to make use of the Poincare isomorphisms between $p$-vector fields and $(\operatorname{dim} M-p)$-exterior forms. The two basic operators to use will be, of course, the interior product $i_{T} \alpha$, where $T$ is a $p$-vector, $\alpha$ is a $q$-form, $p \leqq q$, and the exterior derivative $\mathbf{d}: \Lambda^{p}(M) \rightarrow \Lambda^{p+1}(M)$.

\section{Some formal relations}

Let $E$ and $E^{*}$ be two dual real finite dimensional vector spaces. The duality between $E$ and $E^{*}$ allows to distinguish the following well known (anti)derivation. Let $h \in E$, then we obtain the derivation $i(h)$, or $i_{h}$, in $\Lambda\left(E^{*}\right)$ of degree $(-1)$ (sometimes called substitution/contraction/insertion operator, interior product, algebraic flow) according to [1]:

$$
i(h)\left(x^{* 1} \wedge \cdots \wedge x^{* p}\right)=\sum_{i=1}^{p}(-1)^{(i-1)}\left\langle x^{* i}, h\right\rangle x^{* 1} \wedge \cdots \wedge \hat{x^{* i}} \wedge \cdots \wedge x^{* p} .
$$

Clearly, if $u^{*} \in \Lambda^{p}\left(E^{*}\right)$ and $v^{*} \in \Lambda\left(E^{*}\right)$ then

$$
i(h)\left(u^{*} \wedge v^{*}\right)=\left(i(h) u^{*}\right) \wedge v^{*}+(-1)^{p} u^{*} \wedge i(h) v^{*} .
$$

Also, we get

$$
i(h) u^{*}\left(x_{1}, \ldots, x_{p-1}\right)=u^{*}\left(h, x_{1}, \ldots, x_{p-1}\right),
$$




$$
i(x) \circ i(y)=-i(y) \circ i(x) .
$$

This antiderivation is extended to a mapping $i\left(h_{1} \wedge \cdots \wedge h_{p}\right): \Lambda^{m}\left(E^{*}\right) \rightarrow \Lambda^{(m-p)}\left(E^{*}\right), m \geqq p$, according to

$$
i\left(h_{1} \wedge h_{2} \wedge \cdots \wedge h_{p}\right) u^{*}=i\left(h_{p}\right) \circ \cdots \circ i\left(h_{1}\right) u^{*} .
$$

Note that this extended mapping is not an antiderivation, except for $p=1$.

Clearly, this interior product may be used in "opposite direction", i.e., if $u^{*}$ is 1 -form, then

$$
\begin{gathered}
i\left(u^{*}\right)\left(h_{1} \wedge h_{2} \wedge \ldots \wedge h_{p}\right)=<u^{*}, h_{1}>\left(h_{2} \wedge \ldots \wedge h_{n}\right)-<u^{*}, h_{2}>\left(h_{1} \wedge h_{3} \wedge \ldots \wedge h_{p}\right)+\ldots \\
+(-1)^{(p-1)}<u^{*}, h_{p}>\left(h_{1} \wedge h_{2} \wedge \ldots \wedge h_{p-1}\right) .
\end{gathered}
$$

This mapping is extended to multivectors and exterior forms which are linear combinations: if $\Psi=\Psi_{1}+\Psi_{2}+\ldots$ is an arbitrary multivector on $E$ and $\Phi=\Phi^{1}+\Phi^{2}+\ldots$ is an arbitrary exterior form on $E^{*}$ then $i_{\Psi} \Phi$ is defined as extension by linearity, e.g.,

$$
i\left(\Psi_{1}+\Psi_{2}\right)\left(\Phi^{1}+\Phi^{2}\right)=i\left(\Psi_{1}\right) \Phi^{1}+i\left(\Psi_{1}\right) \Phi^{2}+i\left(\Psi_{2}\right) \Phi^{1}+i\left(\Psi_{2}\right) \Phi^{2} .
$$

The above extension of the interior product allows to extend the Lie derivative of a differential form $\Phi$ along a vector field $X$ to a derivative of $\Phi$ along a multivector field $T$ [2], according to

$$
\mathcal{L}_{T}(\Phi)=d \circ i_{T} \Phi-(-1)^{\operatorname{deg}(T)} i_{T} \circ d \Phi .
$$

If $\mathcal{L}_{T}(\Phi)=0$ this extension allows to consider $T$ as a local symmetry of $\Phi$.

The specialization of these interior products for the cases $i(\Phi) \omega^{*}$ and $i(\beta) \omega$, where $\Phi$ is a multivector, $\omega^{*}=\varepsilon^{1} \wedge \varepsilon^{2} \wedge \ldots \wedge \varepsilon^{n}$ is a volume form in $E^{*}, \beta$ is a $p$-form, and $\omega=e_{1} \wedge e_{2} \wedge \ldots \wedge e_{n}$ is the volume form in $E$, dual to $\left.\omega^{*}:<\omega^{*}, \omega\right\rangle=1$, is well known [1] and used in multilinear algebra and differential topology under the name of Poincare isomorphisms, or Poincare dualities. In fact, we note that the two spaces $\Lambda^{p}(E) \otimes \Lambda^{n}\left(E^{*}\right)$ and $\Lambda^{n-p}\left(E^{*}\right)$ have the same dimension, so, every nonzero $\omega \in \Lambda^{n}\left(E^{*}\right)$ generates isomorphism $D^{p}$, between these two spaces according to $\left(u, \omega^{*}\right) \rightarrow i(u) \omega^{*}$, where $u \in \Lambda^{p}(E)$ is a $p$-vector over $E$. In particular, if $\left\{e_{i}\right\}$ and $\left\{\varepsilon^{j}\right\}$ are dual bases, the corresponding basis elements

$$
e_{\nu_{1}} \wedge \cdots \wedge e_{\nu_{p}}, \quad \nu_{1}<\nu_{2}<\ldots<\nu_{p}
$$

and

$$
\varepsilon^{\nu_{p+1}} \wedge \cdots \wedge \varepsilon^{\nu_{n}}, \quad \nu_{p+1}<\nu_{p+2}<\ldots<\nu_{n},
$$

are connected according to

$$
D^{p}\left(e_{\nu_{1}} \wedge \cdots \wedge e_{\nu_{p}}\right)=(-1)^{\sigma} \varepsilon^{\nu_{p+1}} \wedge \cdots \wedge \varepsilon^{\nu_{n}},
$$

where $\sigma=\sum_{i=1}^{p}\left(\nu_{i}-i\right)$. Also,

$$
\begin{gathered}
D_{p}\left(\varepsilon^{\nu_{1}} \wedge \cdots \wedge \varepsilon^{\nu_{p}}\right)=(-1)^{\sigma} e_{\nu_{p+1}} \wedge \cdots \wedge e_{\nu_{n}}, \\
D_{p}\left(\varepsilon^{\nu_{p+1}} \wedge \cdots \wedge \varepsilon^{\nu_{n}}\right)=(-1)^{p(n-p)+\sigma} e_{\nu_{1}} \wedge \cdots \wedge e_{\nu_{p}} .
\end{gathered}
$$

Clearly, we have

$$
i\left(e_{\nu_{1}} \wedge \cdots \wedge e_{\nu_{p}}\right) D^{p}\left(e_{\nu_{1}} \wedge \cdots \wedge e_{\nu_{p}}\right)=0 .
$$

Also, we note that in this way every subspace $V^{p} \subset E$ leads to defining three other spaces:

$$
\left(V^{p}\right)^{*} \subset E^{*} ; \quad D^{p}\left(V^{p}\right) \subset E^{*} ; \quad D_{p}\left(\left(V^{p}\right)^{*}\right) \subset E,
$$


where

$$
E=V^{p} \oplus\left(D^{p}\left(V^{p}\right)\right)^{*} ; E^{*}=\left(V^{p}\right)^{*} \oplus D^{p}\left(V^{p}\right) .
$$

We remind that these isomorphisms depend on the chosen element $\omega \in \Lambda^{n}\left(E^{*}\right)$, but further in this section we shall omit writing $\omega$ for clarity.

These last two formulas allow to make use of any isomorphism between $E$ and $E^{*}$ for defining isomorphisms $\Lambda^{p}(E) \cong \Lambda^{n-p}(E)$, and $\Lambda^{p}\left(E^{*}\right) \cong \Lambda^{n-p}\left(E^{*}\right)$.

For these isomorphisms and their duals

$$
\begin{aligned}
& D^{p}: \Lambda^{p}(E) \rightarrow \Lambda^{n-p}\left(E^{*}\right), \quad\left(D^{p}\right)^{*}: \Lambda^{n-p}(E) \rightarrow \Lambda^{p}\left(E^{*}\right) \\
& D_{p}: \Lambda^{p}\left(E^{*}\right) \rightarrow \Lambda^{n-p}(E), \quad\left(D_{p}\right)^{*}: \Lambda^{n-p}\left(E^{*}\right) \rightarrow \Lambda^{p}(E),
\end{aligned}
$$

the following relations also hold:

$$
\begin{gathered}
\left(D_{p}\right)^{*}=\left(D^{p}\right)^{-1}=(-1)^{p(n-p)} D_{n-p} ; \quad\left(D^{p}\right)^{*}=\left(D_{p}\right)^{-1}=(-1)^{p(n-p)} D^{n-p} ; \\
D_{n-p} \circ D^{p}=(-1)^{p(n-p)} i d, \quad D^{n-p} \circ D_{p}=(-1)^{p(n-p)} i d,
\end{gathered}
$$

where $i d$ denotes the corresponding identity map. So, up to a sign factor, $D^{p}$ and $D_{n-p}$ are inverse linear isomorphisms. When $T \in \Lambda(E)$ and $\Psi \in \Lambda\left(E^{*}\right)$ are represented by exterior products, for example: $T=u \wedge v, \Psi=\alpha \wedge \beta$, and their degrees are not given, then we replace $D^{p}$ by $\mathfrak{D}$ and $D_{p}$ by $\mathbb{D}$, and the following relations hold

$$
\mathfrak{D}(T)=i_{v} \circ i_{u} \omega, \quad \mathbb{D}(\Psi)=i_{\beta} \circ i_{\alpha} \omega^{*} .
$$

These relations will be used further in Sec.2.4.

We extend now these insertion operators in different direction. Let $E_{1}$ and $E_{2}$ be two real vector spaces with corresponding bases $\left\{e_{i}\right\}, i=1,2, \ldots, \operatorname{dim} E_{1}$ and $\left\{k_{j}\right\}, j=1,2, \ldots, \operatorname{dim} E_{2}, T=\mathfrak{t}^{i} \otimes e_{i}$ be a $E_{1}$-valued $q$-vector, $\Phi=\alpha^{j} \otimes k_{j}$ be a $E_{2}$-valued $p$-form with $q \leq p$, and $\varphi: E_{1} \times E_{2} \rightarrow F$ be a bilinear map into the vector space $F$. Now we define $i_{T}^{\varphi} \Phi \in \Lambda^{p-q}(M, F)$ :

$$
i_{T}^{\varphi} \Phi=i_{\mathfrak{t}^{i}} \alpha^{j} \otimes \varphi\left(e_{i}, k_{j}\right), \quad i=1,2, \ldots, \operatorname{dim}\left(E_{1}\right), j=1,2, \ldots, \operatorname{dim}\left(E_{2}\right) .
$$

Also, if $T_{1}, T_{2}$ are two multivectors and $\alpha, \beta$ are two forms then $(i \otimes i)_{T_{1} \otimes T_{2}}(\alpha \otimes \beta)$ is defined by

$$
(i \otimes i)_{T_{1} \otimes T_{2}}(\alpha \otimes \beta)=i_{T_{1}} \alpha \otimes i_{T_{2}} \beta .
$$

We can define now the $\varphi$-extended Lie derivative. Let $M$ be a $n$-dimensional manifold, $\Phi$ be a $E_{1}$-valued differential $p$-form on $M, T \in \mathfrak{X}^{q}\left(M, E_{2}\right)$ be a $E_{2}$-valued $q$-multivector field on $M$, with $q \leq p$ and $\varphi: E_{1} \times E_{2} \rightarrow F$ be a bilinear map. The $\varphi$-extended Lie derivative

$$
\mathcal{L}_{T}^{\varphi}: \Lambda^{p}\left(M, E_{1}\right) \times \mathfrak{X}^{q}\left(M, E_{2}\right) \rightarrow \Lambda^{p-q+1}(M, F)
$$

is defined as follows [21, p.53]:

$$
\mathcal{L}_{T}^{\varphi}(\Phi)=\mathbf{d} \circ i_{T}^{\varphi} \Phi-(-1)^{\operatorname{deg}(T) \cdot \operatorname{deg}(\mathbf{d})} i_{T}^{\varphi} \circ \mathbf{d} \Phi,
$$

where $\mathbf{d}$ is the exterior derivative on $M$, so, $\operatorname{deg}(\mathbf{d})=1$. This definition suggests to consider the tensor field $T$ as a local $\varphi$-symmetry of the differential form $\Phi$ when $\mathcal{L}_{T}^{\varphi}(\Phi)=0$. 


\section{Pre-metric formulation in the linear case}

Since we are not going to use metric tensors in the cases considered, for more information, including history, analysis and citations, on the so-called pre-metric formulation of electrodynamics, not only in the charge free case, the reader may find in the 2-volume e-book by D.A.Delphenich [3].

\subsection{Pre-relativistic approach}

Let $X$ be a vector field on $\left(\mathbb{R}^{3}, \omega\right)$, where $\omega=d x \wedge d y \wedge d z$ is the usual volume 3-form on $\mathbb{R}^{3}$. The usual interaction partners of any vector field $\mathrm{X}$ are the differential forms $\alpha \in \Lambda\left(\mathbb{R}^{3}\right)$ on the same manifold since the generated by $X$ flow $i_{X} \alpha$ across $\alpha$ may change $\alpha$, i.e., $i_{X} \alpha$ may be not zero: $i_{X} \alpha \neq 0$. In searching for such a differential form partner, our vector field $X$ finds only one that is specially indicated, the volume 3 -form $\omega$. Now the following question arizes: does this flow $i_{X} \omega \neq 0$ change from point to point? Formally this means whether the Lie derivative $L_{X} \omega$ change from point to point along $X$, or not. For this Lie derivative we obtain

$$
L_{X} \omega=\mathbf{d} i_{X} \omega+i_{X} \mathbf{d} \omega=\mathbf{d} i_{X} \omega=\mathbf{d}\left[X^{1} d y \wedge d z-X^{2} d x \wedge d z+X^{3} d x \wedge d y\right]
$$

since $\mathbf{d} \omega=0$ by dimensional reasons. So, if this flow does not change from point to point, the 2-form $i_{X} \omega$ must be closed: $\mathbf{d} i_{X} \omega=0$. As is easily verified, this means that the vector field $X$ has zero divergence:

$$
\mathbf{d} i_{X} \omega=\left[\frac{\partial X^{1}}{\partial x}+\frac{\partial X^{2}}{\partial y}+\frac{\partial X^{3}}{\partial z}\right] d x \wedge d y \wedge d z=(\operatorname{div} X) \cdot \omega=0 \quad \rightarrow \quad \operatorname{div} X=0 .
$$

We specially note that the 2-form $i_{X} \omega$ is closed $: \mathbf{d} i_{X} \omega=0$. Therefore, there exist a class of 1 -forms $\alpha+d f$ such that, locally, we may write $i_{X} \omega=\mathbf{d}(\alpha+d f)$. We conclude:

Every divergence-free vector field on $\left(\mathbb{R}^{3}, \omega\right)$ is naturally connected with some differential 1forms.

Recalling now the two Maxwell equations $\operatorname{div} \mathbf{E}=0, \operatorname{div} \mathbf{B}=0$ we conclude also, that these two vector fields $(\mathbf{E}, \mathbf{B})$ are intrinsically connected with corresponding differential 1-forms, and this connection does NOT require any metric tensor.

However, if the flows generated by $\mathbf{E}$ and $\mathbf{B}$ admit time-change although each of them does not change the volume form $\omega$ ? This question suggests to look more carefully for other possible ways for mutual influence of the two flows generated by $\mathbf{E}$ and $\mathbf{B}$.

According to the above notations the two lineary independent vector fields $(\mathbf{E}, \mathbf{B})$ appear together with their corresponding 2-forms $i_{\mathbf{E}} \omega$ and $i_{\mathbf{B}} \omega$. Moreover, the equations $\operatorname{div} \mathbf{E}=0, \operatorname{div} \mathbf{B}=0$ require these two 2 -forms $\left(i_{\mathbf{E}} \omega, i_{\mathbf{B}} \omega\right)$ to be closed, and so - locally exact. We conclude that there must exist two 1 -forms $\alpha$ and $\beta$ satisfying the equations

$$
i_{\mathbf{E}} \omega=\mathbf{d} \alpha, \quad i_{\mathbf{B}} \omega=\mathbf{d} \beta, \quad i_{\mathbf{E}} \mathbf{d} \alpha=0, \quad i_{\mathbf{B}} \mathbf{d} \beta=0 .
$$

Another suggesting moment in this direction is the seriously ised "curl" operator in Maxwell equations. Making use of the mentioned Poincare isomorphisms between $p$-forms and $(n-p)$ vectors on oriented manifolds, it is easily found that this "curl" operator is strongly connected to the exterior derivative operator $\mathbf{d}$, acting in the graded algebra of differential forms. Explicitly, in the case of our manifold $\left(\mathbb{R}^{3}, \omega\right)$, the restriction $\mathbb{D}$ of the Poincare isomorphism to differential 
2 -forms of the kind $\mathbf{d} \alpha$, where $\alpha$ is arbitrary 1-form, maps $\mathbf{d} \alpha$ to a unique vector field of the kind $\operatorname{curl}(\mathbf{V})$ :

$$
\mathbb{D}(\mathbf{d} \alpha)=\operatorname{curl}(\mathbf{V}), \quad \text { or, } \quad \mathfrak{D}(\operatorname{curl}(\mathbf{V}))=\mathbf{d} \alpha,
$$

and the components of $\mathbf{d} \alpha$ in the basis $(d x \wedge d y, d x \wedge d z, d y \wedge d z)$ coincide with the components of $\operatorname{curl}(\mathbf{V})$ in the basis $\left(\frac{\partial}{\partial x}, \frac{\partial}{\partial y}, \frac{\partial}{\partial z}\right)$. Of course, most easily this can be achieved if the components of $\alpha$ and the components of $\mathbf{V}$ are the same in the corresponding dual bases $(d x, d y, d z) \leftrightarrow\left(\frac{\partial}{\partial x}, \frac{\partial}{\partial y}, \frac{\partial}{\partial z}\right)$.

Also, Poincare isomorphism generates two bivectors $\bar{H}$ and $\bar{K}$ according to

$$
\alpha=i_{\bar{H}} \omega, \quad \beta=i_{\bar{K}} \omega
$$

All this suggests to look for realizable $\mathbf{E} \leftrightarrow \mathbf{B}$ time-interaction making use of $\alpha$ and $\beta$.

In Maxwell theory time is introduced as external to the coordinates $(x, y, z)$ parameter, denoted by $t$, so, the time differentiation of tensor fields on $\mathbb{R}^{3}$ does NOT change the tensor nature of the object. It seems that $i_{\mathbf{E}} \omega$ and $i_{\mathbf{B}} \omega$ may have a chance to find their corresponding "curl" partners paying appropriate respect to their own time derivatives. This suggests to answer positively to the question "do available non-zero time derivatives of $(\mathbf{E}, \mathbf{B})$ find way for realization through the 1form partners". As is well known, classical charge free electrodynamics, as represented by Maxwell equations, gives a positive answer to this question. In our approach it looks as follows.

The closed nature of $i_{\mathbf{E}} \omega$ and $i_{\mathbf{B}} \omega$ and the above consideration and notations allow in the corresponding bases $\left(\frac{\partial}{\partial x}, \frac{\partial}{\partial y}, \frac{\partial}{\partial z}\right)$ and $(d x \wedge d y, d x \wedge d z, d y \wedge d z)$ to write down

$$
\mathfrak{D}(\operatorname{curl}(\mathbf{E}))=\mathbf{d} \alpha, \quad \text { or, } \quad \mathbb{D}(\mathbf{d} \alpha)=\operatorname{curl}(\mathbf{E}),
$$

and

$$
\mathfrak{D}(\operatorname{curl}(\mathbf{B}))=\mathbf{d} \beta, \quad \text { or, } \quad \mathbb{D}(\mathbf{d} \beta)=\operatorname{curl}(\mathbf{B}) .
$$

Now, in order to model mutual and time-realizable interaction between $\mathbf{E}$ and $\mathbf{B}$ making help of $\alpha$ and $\beta$, Maxwell consistency condition requires

$$
\frac{1}{c} \frac{\partial}{\partial t} i_{\mathbf{E}} \omega=\mathbf{d} \beta, \quad \frac{1}{c} \frac{\partial}{\partial t} i_{\mathbf{B}} \omega=-\mathbf{d} \alpha
$$

where $c$ denotes an invariant constant velocity, characterizing the translational propagation of the electromagnetic field object considered. Hence, equations (4) formally introduce a kind of crossconnection between the divergence-free nature of $(\mathbf{E}, \mathbf{B})$ and the external nature of their possible time dependence.

So, the content of this positive answer physically means that time derivatives of $(\mathbf{E}, \mathbf{B})$ interchange their 1-form partners: a time change of $\mathbf{E}$ requires appropriate local spatial change of the 1-form partner $\beta$ of $\mathbf{B}$, and a time change of $\mathbf{B}$ requires appropriate local spatial change of the 1-form partner $\alpha$ of $\mathbf{E}$. We note that, from formal viewpoint, this partnership makes use of the invariant operator exterior derivative, so the partnership is real, and the introduced cross-connection of this reality with the corresponding time derivatives allows to consider the time dependent electromagnetic field objects as real ones.

Recall now the concept of local helicity generated by any 1 -form $\eta$ on $\mathbb{R}^{3}$, it is defined as the 3 -form $\eta \wedge \mathbf{d} \eta$ and carries information about the rotational properties of the flow generated by a divergence-free vector field $X$ that is euclidean metric image of $\eta: \eta_{i}=g_{i j} X^{j}$, or $X^{i}=g^{i j} \eta_{j}$, where $g$ denotes the euclidean metric tensor on $\mathbb{R}^{3}$. In fact, $\eta \wedge \mathbf{d} \eta=g(X, \operatorname{curl} X) . \omega$. 
The above equations (4) determine time-partnership respectively between $(\mathbf{E}, \beta)$ and between $(\mathbf{B}, \alpha)$. This suggests to show appropriate respect to the corresponding cross helicities, given by $\alpha \wedge \mathbf{d} \beta$ and $\beta \wedge \mathbf{d} \alpha$. From classical vector analysis we have the relation

$$
L_{\mathbf{E} \times \mathbf{B}} \omega=[\mathbf{B} \cdot \operatorname{curl}(\mathbf{E})-\mathbf{E} \cdot \operatorname{curl}(\mathbf{B})] \omega=\operatorname{div}(\mathbf{E} \times \mathbf{B}) . \omega .
$$

The corresponding Poynting relation in Maxwell theory suggests now the following. Since our field object will propagate in the 3 -space, then at the points where the field functions are not zero at the moment $t$, generally speaking, there must be expected time changes of the important local characteristics of the object at these points. Trying to present these changes without making use of a metric, we can make use of the above introduced cross helicities as follows:

$$
\beta \wedge \mathbf{d} \alpha-\alpha \wedge \mathbf{d} \beta=-\frac{\partial}{\partial \xi}\left(\frac{<\alpha, \mathbf{E}>+<\beta, \mathbf{B}>}{2}\right) . \omega, \xi=c t .
$$

We form now the following $(1,1)$ tensor:

$$
\mathbb{T}=\alpha \otimes \mathbf{E}+\beta \otimes \mathbf{B}-\frac{1}{2}(<\alpha, \mathbf{E}>+<\beta, \mathbf{B}>) i d_{T \mathbb{R}^{3}} .
$$

Obviously, this tensor represents a metric-free form of Maxwell stress tensor.

A more careful look at $\mathbb{T}$ naturally sets the question "weather the two vector fields $\mathbf{E}$ and $\mathbf{B}$, which may be considered as constituents of $\mathbb{T}$, define eigen directions of $\mathbb{T}$ at every point". The corresponding to this question equations read:

$$
i_{\mathbf{E}} \mathbb{T}=\lambda_{1} . \mathbf{E}, \quad i_{\mathbf{B}} \mathbb{T}=\lambda_{2} . \mathbf{B} .
$$

Since $\mathbf{E}$ and $\mathbf{B}$ are lineary independent, it is elementary to see that these two relations hold only if

$$
<\alpha, \mathbf{B}>=0, \quad<\beta, \mathbf{E}>=0
$$

So, we have two more algebraic equations connecting $(\mathbf{E}, \mathbf{B})$ with $(\alpha, \beta)$.

Finally, the mutually dependent vector fields $(\mathbf{E}, \mathbf{B})$ define the bi-vector $\mathbf{E} \wedge \mathbf{B}$, and a natural algebraic characteristic of the assumed dependence between $(\mathbf{E}, \mathbf{B})$ and $(\alpha, \beta)$ appears to be the flow of of $\mathbf{E} \wedge \mathbf{B}$ across the 2-form $\alpha \wedge \beta$. In view of the above last relations for this flow we obtain

$$
i_{\mathbf{E} \wedge \mathbf{B}}(\alpha \wedge \beta)=i_{\mathbf{B}} \circ i_{\mathbf{E}}(\alpha \wedge \beta)=<\alpha, \mathbf{E}><\beta, \mathbf{B}>-<\alpha, \mathbf{B}><\beta, \mathbf{E}>=<\alpha, \mathbf{E}><\beta, \mathbf{B}>.
$$

We may assume now the following way to relate this nonlinear flow with the linear flow of $\mathbf{E}$ across $\alpha$, given by $\langle\alpha, \mathbf{E}>$, and of $\mathbf{B}$ across $\beta$, given by $\langle\beta, \mathbf{B}>$ :

$$
i_{\mathbf{E} \wedge \mathbf{B}}(\alpha \wedge \beta)=\left[\frac{<\alpha, \mathbf{E}>+<\beta, \mathbf{B}>}{2}\right]^{2} .
$$

From these last two algebraic relations it will follow

$$
<\alpha, \mathbf{E}>^{2}-2<\alpha, \mathbf{E}>.<\beta, \mathbf{B}>+<\beta, \mathbf{B}>^{2}=(<\alpha, \mathbf{E}>-<\beta, \mathbf{B}>)^{2}=0,
$$

i.e., $<\alpha, \mathbf{E}>=<\beta, \mathbf{B}>$.

\section{Remark.}

Considering the flow of $\mathbf{E} \wedge \mathbf{B}$ across $\omega$ we get the 1-form

$$
\theta=i_{\mathbf{E} \wedge \mathbf{B}} \omega
$$


Since $i_{\mathbf{E} \wedge \mathbf{B}}=i_{\mathbf{B}} \circ i_{\mathbf{E}}$, we get $i_{\mathbf{E}} \theta=i_{\mathbf{B}} \theta=0$, therefore, this 1-form $\theta$ may carry information about the integrability of the 2-dimensional distribution $\Gamma$ on $\mathbb{R}^{3}$ defined by $\mathbf{E}$ and $\mathbf{B}$. It seems reasonable to admit that in some cases $\Gamma$ may be integrable which formally means that $\theta$ must satisfy the equation

$$
\mathbf{d} \theta \wedge \theta=0,
$$

for example, when time stable and spatially finite solutions are under consideration. In the considered linear case it is hard to believe that such solutions exist at all, but let it be in sight.

In this way we have 11 equations for the 12 components of $\mathbf{E}, \mathbf{B}, \alpha, \beta$. They read:

$$
\begin{gathered}
L_{\mathbf{E}} \omega=0, \quad L_{\mathbf{B}} \omega=0, \quad \frac{\partial}{\partial \xi} i_{\mathbf{E}} \omega=\mathbf{d} \beta, \quad \frac{\partial}{\partial \xi} i_{\mathbf{B}} \omega=-\mathbf{d} \alpha, \\
i_{\mathbf{E}} \mathbb{T}=\lambda_{1} . \mathbf{E}, \quad i_{\mathbf{B}} \mathbb{T}=\lambda_{2} . \mathbf{B}, \quad<\alpha, \mathbf{E}>=<\beta, \mathbf{B}>, \quad \xi=c t .
\end{gathered}
$$

These equations do not use any metric, so, this approach may represent the nonrelativistic view on so called pre-metric formulation of charge free Maxwell equations.

\subsection{Relativistic approach}

The relativistic approach to electrodynamic phenomena seriously uses the assumption that electromagnetic field objects propagate in free space with constant speed $c$, where "constant" means "the same with respect to any reference frame", i.e., with respect to any free mass body. This brought the necessity to introduce the notion that time-measuring depends on the reference frame with respect to which the time-measuring system does not move. In this way the fourth coordinate $\xi=c t, t$ is the measured time interval, was introduced, the 3 -space $\mathbb{R}^{3}$ was extended to $\mathbb{R}^{4}$, and the 3-space volume form $\omega_{3}=d x \wedge d y \wedge d z$ was extended to 4-spacetime volume form $\omega$ :

$$
\omega=\omega_{3} \wedge d \xi=d x \wedge d y \wedge d z \wedge d \xi
$$

where $d \xi=c d t$.

The above mentioned Maxwell consistency condition suggests the following extension of the two 2 -forms $i_{\mathbf{E}} \omega_{3}$ and $i_{\mathbf{B}} \omega_{3}$ to two spacetime 2-forms:

$F=i_{\mathbf{B}} \omega_{3}+\alpha \wedge d \xi=B^{3} d x \wedge d y-B^{2} d x \wedge d z+B^{1} d y \wedge d z+\alpha_{1} d x \wedge d \xi+\alpha_{2} d y \wedge d \xi+\alpha_{3} d z \wedge d \xi$, $G=i_{\mathbf{E}} \omega_{3}-\beta \wedge d \xi=E^{3} d x \wedge d y-E^{2} d x \wedge d z+E^{1} d y \wedge d z-\beta_{1} d x \wedge d \xi-\beta_{2} d y \wedge d \xi-\beta_{3} d z \wedge d \xi$, where $\mathbf{E}^{i}=\alpha_{i}$, and $\mathbf{B}^{i}=\beta_{i}, i=1,2,3$. Making use now of the new volume 4-form $\omega$, the corresponding bi-vector fields $\bar{F}$ and $\bar{G}$ are defined by

$$
F=i_{\bar{G}} \omega, \quad G=-i_{\bar{F}} \omega
$$

Explicitly,

$$
\begin{aligned}
& \bar{F}=\beta_{3} \frac{\partial}{\partial x} \wedge \frac{\partial}{\partial y}-\beta_{2} \frac{\partial}{\partial x} \wedge \frac{\partial}{\partial z}+\beta_{1} \frac{\partial}{\partial y} \wedge \frac{\partial}{\partial z}-E^{1} \frac{\partial}{\partial x} \wedge \frac{\partial}{\partial \xi}-E^{2} \frac{\partial}{\partial y} \wedge \frac{\partial}{\partial \xi}-E^{3} \frac{\partial}{\partial z} \wedge \frac{\partial}{\partial \xi} \\
& \bar{G}=\alpha_{3} \frac{\partial}{\partial x} \wedge \frac{\partial}{\partial y}-\alpha_{2} \frac{\partial}{\partial x} \wedge \frac{\partial}{\partial z}+\alpha_{1} \frac{\partial}{\partial y} \wedge \frac{\partial}{\partial z}+B^{1} \frac{\partial}{\partial x} \wedge \frac{\partial}{\partial \xi}+B^{2} \frac{\partial}{\partial y} \wedge \frac{\partial}{\partial \xi}+B^{3} \frac{\partial}{\partial z} \wedge \frac{\partial}{\partial \xi}
\end{aligned}
$$

The following relations hold:

$$
<F, \bar{F}>=<\beta, \mathbf{B}>-<\alpha, \mathbf{E}>, \quad<G, \bar{G}>=<\alpha, \mathbf{E}>-<\beta, \mathbf{B}>,
$$




$$
<F, \bar{G}>=2<\alpha, \mathbf{B}>, \quad<G, \bar{F}>=2<\beta, \mathbf{E}>,
$$

so, $<F, \bar{F}>=-<G, \bar{G}>$.

Now, since $\mathbf{d} \omega=0$, the zero value of the mentioned extension of the Lie derivative (3) gives the equations

$$
\mathcal{L}_{\bar{F}} \omega=\mathbf{d} i_{\bar{F}} \omega=-\mathbf{d} G=0, \quad \mathcal{L}_{\bar{G}} \omega=\mathbf{d} i_{\bar{G}} \omega=\mathbf{d} F=0,
$$

which reproduce Maxwell charge free equations (5) in a metric-free way, i.e., the two 2 -forms $F, G$ are closed: $\mathbf{d} F=0, \mathbf{d} G=0$. In other words, the flows of the bi-vectors $\bar{F}$ and $\bar{G}$ do NOT change the volume 4 -form $\omega$.

If $C_{2}^{2}$ is the contraction operator with respect to the second member, then the $(1,1)$-stressenergy-momentum tensor $\mathbb{T}_{(F, G)}$ admits the following coordinate-free and metric-free form

$$
\mathbb{T}=-\frac{1}{2}\left[C_{2}^{2}(F \otimes \bar{F})+C_{2}^{2}(G \otimes \bar{G})\right] .
$$

Another look at $\mathbb{T}_{(F, G)}$ is the following. Let $X$ be an arbitrary vector field on $\mathbb{R}^{4}$ and $\theta$ be an arbitrary 1-form on $\mathbb{R}^{4}$. We can form now $i_{X} F=X^{\sigma} F_{\sigma \nu} d x^{\nu}$ and $i_{\theta} \bar{F}=\theta_{\sigma} \bar{F}^{\sigma \nu} \frac{\partial}{\partial x^{\nu}}$. The tensor $\mathbb{T}_{(F, G)}$ may now be defined in the following way:

$$
\mathbb{T}(X, \theta)=-\frac{1}{2} \operatorname{tr}\left[i_{X} F \otimes i_{\theta} \bar{F}+i_{X} G \otimes i_{\theta} \bar{G}\right] .
$$

In this way we get a relativistic pre-metric view on Maxwell charge-free equations: no Minkowski pseudometric, no Hodge "*". (detailed comments see in [3]).

\section{Preliminary notes on non-linearization}

Nonlinearizations of Maxwell charge free electrodynamics the reader may find in $[4], \ldots,[17]$ and $[18], \ldots,[21]$.

Passing to nonlinear field equations we begin with two appropriate examples [21, p.370-371;22].

The examples consider the nonlinear equations defining the so called autoparallel time-like and null vector fields with respect to a given connection, restricting to the simple case of Levi-Civita connection $\Gamma=\left(\Gamma_{\mu \nu}^{\sigma}\right)$ on Minkowski space-time $\left(\mathbb{R}^{4}, \eta\right)$.

Recalling the definition of autoparallel vector field: $\nabla_{X} X=0$, or $i_{X} \nabla X=0$, it is interesting to note that this nonlinear system of equations admits $(3+1)$-soliton-like, even spatially finite, solutions on Minkowski space-time.

In fact, in canonical coordinates $\left(x^{1}, x^{2}, x^{3}, x^{4}\right)=(x, y, z, \xi=c t)$ we have $\Gamma_{\mu \nu}^{\sigma}=0$, and let $u^{\mu}=\left(0,0, \pm \frac{v}{c} f, f\right)$, be the components of the time-like vector field $u, \eta(u, u)>0$, where $0<v=$ const $<c$, and $c$ is the velocity of light, so $\frac{v}{c}<1$ and $\eta(u, u)=\left(1-\frac{v^{2}}{c^{2}}\right) f^{2}>0$. Then every function $f$ of the kind

$$
f(x, y, z, \xi)=f\left(x, y, \alpha .\left(z \mp \frac{v}{c} \xi\right)\right), \alpha=\text { const }, \quad \text { for example } \quad \alpha=\frac{1}{\sqrt{1-\frac{v^{2}}{c^{2}}}},
$$

defines a solution.

If $\eta(u, u)=0$ then the equations are equivalent to $u^{\mu}(\mathbf{d} \tilde{\eta}(u))_{\mu \nu}=0$, where $\mathbf{d}$ is the exterior derivative. In fact, since the connection used is riemannian, we have $0=\nabla_{\mu} \frac{1}{2}\left(u^{\nu} u_{\nu}\right)=u^{\nu} \nabla_{\mu} u_{\nu}$, so 
the relation $u^{\nu} \nabla_{\nu} u_{\mu}-u^{\nu} \nabla_{\mu} u_{\nu}=0$ holds and is obviously equal to $u^{\mu}(\mathbf{d} u)_{\mu \nu}=0$. The soliton-like solution is defined by $u^{\mu}=(0,0, \pm f, f)$ where the function $f$ is of the form

$$
f(x, y, z, \xi)=f(x, y, z \mp \xi) .
$$

Clearly, for every autoparallel vector field $u$ (or one-form $u$ ) there exists a canonical coordinate system on the Minkowski space-time, in which $u$ takes such a simple form: $u^{\mu}=(0,0, \alpha f, f), \alpha=$ const. The dependence of $f$ on the three spatial coordinates $(x, y, z)$ is arbitrary, so it is allowed to be chosen soliton-like and, even, spatially finite.

So, although the trajectories of these autoparallel vector fields are straight lines and in this sense are naturally considered as appropriate description of free point-like objects, the field nature of these vector fields deserves corresponding attention and respect as a generator of $(3+1)$-spatially finite solutions. Moreover, these equations suggest one possible way to find appropriate nonlinearization in other more complex cases: having the mathematical image $\Phi$ of the physical object of interest and its change $\nabla \Phi$, compute the flow $\mathfrak{F}$ of $\Phi$ across $\nabla \Phi$, and put this flow equal to zero. This means that the change $\nabla \Phi$ is admissible for $\Phi$, i.e., the physical object considered, appropriately changes under the action of external fields of different nature in order to survive. Of course, if the object considered consists of several interacting subsystems, then the external and the mutual among the subsystems interaction has to be correspondingly identified and formally represented in order to get the right entire and complete picture of the object's appearance and behavior.

In the frame of such approach we are going to nonlinearize Maxwell charge free equations in a pre-metric way, trying to build spatially finite image of a photon-like object, consisting of two interacting subsystems, and the constituents of these two subsystems to be formally represented in terms of already introduced four fields $(\mathbf{E}, \mathbf{B} ; \alpha, \beta)$.

The first step to nonlinearization considers the case, of course, of non-zero divergences of $\mathbf{E}$ and $\mathbf{B}$, i.e., $\mathbf{d} i_{\mathbf{E}} \omega_{3} \neq 0, \quad \mathbf{d} i_{\mathbf{B}} \omega_{3} \neq 0$, in general, and available local interaction between the flows generated by the two vector fields $(\mathbf{E}, \mathbf{B})$, not ignoring, of course, the participation of their 1-form companions $(\alpha, \beta)$. Note that, in the linear case, the basic constituents of the situation were these four objects, and no definite local time-stable subsystems created by them were defined and used. Our view on available intrinsic interaction, i.e., local stress-energy-momentum exchange, inside an electromagnetic field object is based on the following presumption:

Existence of recognizable time-stable subsystems of the object considered, which subsystems are able to carry and exchange stress-energy-momentum, is required.

Therefore, our first step should be to formally identify and further recognize such subsystems. Our approach is based on the hypothesis that the subsystems are just two. As formal constituents of these two subsystems we choose the mathematical objects $(\mathbf{E}, \mathbf{B} ; \alpha, \beta)$, where, as we mentioned, $(\mathbf{E}, \mathbf{B})$ are allowed now to have NON-zero divergences, so, in general, $i_{\mathbf{E}} \mathbf{d} \alpha \neq 0, i_{\mathbf{B}} \mathbf{d} \beta \neq 0$, $i_{\mathbf{E}} \mathbf{d} \beta \neq 0$ and $i_{\mathbf{B}} \mathbf{d} \alpha \neq 0$.

\section{Pre-metric formulation in the non-linear case}

\subsection{Static case}

In this case in order to follow the above given view, we consider defined on $\mathbb{R}^{3}$ vector-valued objects, in terms of which the mathematical images of two subsystems will be created. Since the subsystems are two, our objects will take values in a 2-dimensional real vector space denoted further by $V$, and equipped with a basis $\left(e_{1}, e_{2}\right)$. 
The first subsystem $(\Omega, \bar{\Omega})$ consists of the following two $V$-valued objects:

$$
\Omega=\alpha \otimes e_{1}+\beta \otimes e_{2}, \quad \bar{\Omega}=\mathbf{E} \otimes e_{1}+\mathbf{B} \otimes e_{2} .
$$

Recalling now that the two 1-forms $(\alpha, \beta)$ have their bi-vector $\omega_{3}$-images $\bar{H}$ and $\bar{K}$ according to $\alpha=i_{\bar{H}} \omega_{3}, \quad \beta=i_{\bar{K}} \omega_{3}, \quad$ we define the second subsystem $(\Sigma, \bar{\Sigma})$ as follows:

$$
\Sigma=-i_{\mathbf{B}} \omega_{3} \otimes e_{1}+i_{\mathbf{E}} \omega_{3} \otimes e_{2}, \quad \bar{\Sigma}=-\bar{K} \otimes e_{1}+\bar{H} \otimes e_{2} .
$$

Note that while $\Omega$ is $V$-valued 1-form, $\Sigma$ is $V$-valued 2-form. Correspondingly, $\bar{\Omega}$ is $V$-valued vector field, and $\bar{\Sigma}$ is $V$-valued bi-vector field.

In this static situation, i.e., there are no running processes, any admissible stress-energy exchange between the two subsystems should be realized as dynamical stress equilibrium, so, it must have the following special property: it must be simultaneous and in equal quantities, otherwise the required static situation will be disturbed. Formally this means that any local stress-energy loss/gain of $(\Omega, \bar{\Omega})$ is simultaneously compensated by corresponding local stress-energy gain/loss of $(\Sigma, \bar{\Sigma})$, and vice versa. We could say that our two subsystems demonstrate stable local stress equilibrium. These preliminary remarks suggest: such stress-equilibrium situations need NOT available nonzero interaction stress-energy, which corresponds to the Maxwell stress-energy tensor which does not contain interaction stress-energy between the two constituents $\mathbf{E}$ and $\mathbf{B}$ : the full stress-energy is the sum of the stress-energies carried by the two constituents:

$$
\begin{gathered}
M_{j}^{i}(\mathbf{E}, \mathbf{B})=M_{j}^{i}(\mathbf{E})+M_{j}^{i}(\mathbf{B})=\left(\mathbf{E}^{i} \mathbf{E}_{j}-\frac{1}{2} \mathbf{E}^{2} \delta_{j}^{i}\right)+\left(\mathbf{B}^{i} \mathbf{B}_{j}-\frac{1}{2} \mathbf{B}^{2} \delta_{j}^{i}\right) \\
=\frac{1}{2}\left[\mathbf{E}^{i} \mathbf{E}_{j}+\left(i_{\mathbf{E}} \omega_{3}\right)^{i k}\left(i_{\mathbf{E}} \omega_{3}\right)_{k j}+\mathbf{B}^{i} \mathbf{B}_{j}+\left(i_{\mathbf{B}} \omega_{3}\right)^{i k}\left(i_{\mathbf{B}} \omega_{3}\right)_{k j}\right] .
\end{gathered}
$$

The corresponding equations, defining local stress equilibrium, must represent the following picture: each subsystem keeps locally its stress-energy and the possible changes are mutually compensated. Assuming that these changes are real, their corresponding formal expressions should have tensor nature, so, the most appropriate formal expressions seem to be given by $\mathbf{d} \Omega$ and $\mathbf{d} \Sigma$. The corresponding flows should also pay respect to the vector-valued nature of the formal images of the object's subsystems. These specificities of the stress-energy exchange suggest: the stress-energy balance between the two subsystems to make use of the " $\vee$ "-extension (2) of the interior product as follows:

$$
i_{\bar{\Omega}}^{\vee} \mathbf{d} \Omega=-i \stackrel{\vee}{\vee} \mathbf{d} \Sigma
$$

Our view is that equation (11) adequately corresponds to the fact that there is NO interaction stress in $M_{j}^{i}(\mathbf{E}, \mathbf{B})$ : the whole stress should be carried by $(\Omega, \bar{\Omega})$ and $(\Sigma, \bar{\Sigma})$, and the hidden "dynamical" aspect of this equilibrium is adequately represented by the " $\vee$ "-extension of the interior product. Recalling that $\mathbf{d} i_{X} \omega_{3}=(\operatorname{div} X) \cdot \omega_{3}$ and the above notations: $\alpha=i_{\bar{H}} \omega_{3}$ and $\beta=i_{\bar{K}} \omega_{3}$ we obtain

$$
\begin{gathered}
\mathbf{d} \Omega=\mathbf{d} \alpha \otimes e_{1}+\mathbf{d} \beta \otimes e_{2}, \quad \mathbf{d} \Sigma=-\mathbf{d} i_{\mathbf{B}} \omega_{3} \otimes e_{1}+\mathbf{d} i_{\mathbf{E}} \omega_{3} \otimes e_{2}, \\
i_{\bar{\Omega}}^{\vee} \mathbf{d} \Omega=i_{\mathbf{E}} \mathbf{d} \alpha \otimes e_{1} \vee e_{1}+i_{\mathbf{B}} \mathbf{d} \beta \otimes e_{2} \vee e_{2}+\left(i_{\mathbf{E}} \mathbf{d} \beta+i_{\mathbf{B}} \mathbf{d} \alpha\right) \otimes e_{1} \vee e_{2}, \\
i_{\bar{\Sigma}} \mathbf{d} \Sigma=\beta . \operatorname{div}(\mathbf{B}) \otimes e_{1} \vee e_{1}+\alpha . \operatorname{div}(\mathbf{E}) \otimes e_{2} \vee e_{2}-[\beta . \operatorname{div} \mathbf{E}+\alpha . \operatorname{div} \mathbf{B}] \otimes e_{1} \vee e_{2} .
\end{gathered}
$$

Hence, the balance relation (11) leads to

$$
\begin{aligned}
& i_{\mathbf{E}} \mathbf{d} \alpha+\operatorname{div}(\mathbf{B}) \cdot \beta=0, \\
& i_{\mathbf{B}} \mathbf{d} \beta+\operatorname{div}(\mathbf{E}) \cdot \alpha=0, \\
& i_{\mathbf{E}} \mathbf{d} \beta+i_{\mathbf{B}} \mathbf{d} \alpha-\operatorname{div}(\mathbf{E}) \cdot \beta-\operatorname{div}(\mathbf{B}) \cdot \alpha=0 .
\end{aligned}
$$


The nonzero values of $\operatorname{div} \mathbf{E}, \operatorname{div} \mathbf{B}, i_{\mathbf{E}} \mathbf{d} \alpha, i_{\mathbf{B}} \mathbf{d} \beta, i_{\mathbf{E}} \mathbf{d} \beta$ and $i_{\mathbf{B}} \mathbf{d} \alpha$ provide possible interaction between the two subsystems formally represent by $(\Omega, \bar{\Omega})$ and $(\Sigma, \bar{\Sigma})$, so, the two subsystems acquire status of interacting subsystems of a larger stress-balanced system, i.e., being in the state of dynamical equilibrium.

\subsection{Time dependent case}

First we note that introducing time is considered here as a quantitative comparison of the courses of two physically independent processes, the one of which we call referent, e.g., the progress of appropriate watch, then the other one attains significance of parametrized process.

Hence, we have to specially note that the time parameter $t$ used in this subsection we continue to consider as external to the spatial coordinates $(x, y, z)$ parameter, and the corresponding referent process must NOT influence the parametrized process. The main formal consequence of this consideration is, as we mentioned earlier, that time-derivatives do not change the tensor nature of the $t$-differentiated object.

Naturally, from physical viewpoint, any observed time change of the above discussed stress balance in the static case should presume corresponding influence, leading to its violation, and, of course, to violation of its formal representation - relation (11). Physically it may be expected the electromagnetic field object described to survive through some kind of time "pulsating" at the space points, or through a propagation as a whole in the 3-space, or, both. So: the local static balance should be replaced by an appropriate intrinsically compatible local dynamical and time dependent balance. Hence, in order to survive, our object must be able to generate appropriate spatial changes inside any spatial area that it occupies at any moment of its existence.

To this time-dependence of the behavior of our electromagnetic field object we are going to give formal description by means of finding appropriate change of the static balance equation (11).

Equation (11) formally postulates equivalence between two vector valued 1-forms, so, any introduced influence object, representing how the new time-dependent balance would look like, is expected, formally, also to be vector valued 1-form, containing appropriately first order $(\xi=c t)$ derivative(s) and valued in the same vector space. This allows a natural return to the static balance equation through setting this new object equal to zero.

Also, since the available spatial differential operators in (11) are just of first order, it seems natural the corresponding formal influence object to contain time derivatives of not higher than first order. Clearly, in view of the flow nature of the objects across their own spatially differentiated objects in the static relation (11), the influence object is expected to express formally also a flow, but a flow across time differentiated object. Moreover, it should be expected also this time dependence to generate direct mutual influence between the two now time-dependent subsystems. Finally, since time derivation must not change the tensor nature of the differentiated object, and since $\Omega$ is 1-form, then the 2-form $\Sigma$ is the natural candidate to be $\xi$-differentiated, and the " $\vee$ "-flow of $\bar{\Omega}$ across the $\xi$-differentiated $\Sigma$ will give vector valued 1-form, which naturally appears as appropriate formal measure of the local stress-energy time-exchange. So, we may write

$$
i_{\bar{\Omega}}^{\vee} \mathbf{d} \Omega+i_{\bar{\Sigma}}^{\vee} \mathbf{d} \Sigma=i \frac{\vee}{\vee} \frac{\partial}{\partial \xi} \Sigma .
$$

This equation (12) gives the following three equations

$$
\begin{gathered}
i_{\mathbf{E}} \mathbf{d} \alpha+(\operatorname{div} \mathbf{B}) \cdot \beta=-i_{\left(\frac{\partial \mathbf{B}}{\partial \xi} \wedge \mathbf{E}\right)} \omega_{3}, \\
i_{\mathbf{B}} \mathbf{d} \beta+(\operatorname{div} \mathbf{E}) \cdot \alpha=i_{\left(\frac{\partial \mathbf{E}}{\partial \xi} \wedge \mathbf{B}\right)} \omega_{3}
\end{gathered}
$$




$$
i_{\mathbf{E}} \mathbf{d} \beta+i_{\mathbf{B}} \mathbf{d} \alpha-(\operatorname{div} \mathbf{E}) \cdot \beta-(\operatorname{div} \mathbf{B}) \cdot \alpha=i_{\left(\frac{\partial \mathbf{E}}{\partial \xi} \wedge \mathbf{E}\right)} \omega_{3}-i_{\left(\frac{\partial \mathbf{B}}{\partial \xi} \wedge \mathbf{B}\right)} \omega_{3} .
$$

\subsection{Space-time representation}

In the frame of the space-time view on physical processes the introduced variable $\xi=c t$ is no more independent on the choice of physical frames with respect to which we introduce spatial coordinates and write down time-dependent formal relations. Now $\xi$ is considered as appropriate coordinate, it generates local coordinate base vector $\frac{\partial}{\partial \xi}$ and corresponding co-vector (or 1-form) $d \xi,\left\langle d \xi, \frac{\partial}{\partial \xi}\right\rangle=1$. So, the 3-volume $\omega_{3}=d x \wedge d y \wedge d z$ naturally extends to the 4 -volume $\omega=d x \wedge d y \wedge d z \wedge d \xi$ on $\mathbb{R}^{4}$. Our purpose now is to find appropriate 4-dimensional balance law, suggested by the previous balance laws formally given by equations (11),(12).

Recall our two basic objects: the vector valued differential 1-form $\Omega=\alpha \otimes e_{1}+\beta \otimes e_{2}$ and the vector valued differential 2-form $\Sigma=-i_{\mathbf{B}} \omega_{3} \otimes e_{1}+i_{\mathbf{E}} \omega_{3} \otimes e_{2}$, been defined entirely in terms of objects previously introduced on $\mathbb{R}^{3}$. We want now these objects to depend on $\xi$ as they depend on the spatial coordinates, so to be appropriately extended to objects on $\mathbb{R}^{4}$.

Now, the 4 th dimension $\xi$ generates the coordinate 1 -form $d \xi$, so, the vector valued 1 -form $\Omega$ turns to $d \xi$ for help to extend to a 2 -form on $\mathbb{R}^{4}$, which is done in the simplest way: $\Omega \rightarrow \Omega \wedge d \xi$. We are in position now to consider the difference $\Omega \wedge d \xi-\Sigma$.

$$
\begin{aligned}
\Omega \wedge d \xi-\Sigma & =(\alpha \wedge d \xi) \otimes e_{1}+(\beta \wedge d \xi) \otimes e_{2}+i_{\mathbf{B}} \omega_{3} \otimes e_{1}-i_{\mathbf{E}} \omega_{3} \otimes e_{2} \\
& =\left(i_{\mathbf{B}} \omega_{3}+\alpha \wedge d \xi\right) \otimes e_{1}-\left(i_{\mathbf{E}} \omega_{3}-\beta \wedge d \xi\right) \otimes e_{2}
\end{aligned}
$$

In this way we get two differential 2 -forms on $\mathbb{R}^{4}$ naturally recognized by the basis vectors $\left(e_{1}, e_{2}\right)$ of the external vector space $V$ :

$$
F=i_{\mathbf{B}} \omega_{3}+\alpha \wedge d \xi \quad \text { and } \quad G=i_{\mathbf{E}} \omega_{3}-\beta \wedge d \xi .
$$

These two 2-forms we consider further as vector components of one $V$-valued 2-form $\boldsymbol{\Omega}$ :

$$
\boldsymbol{\Omega}=F \otimes e_{1}+G \otimes e_{2} .
$$

In order to define corresponding flow, as we did it in previous subsections, we have to construct $\overline{\boldsymbol{\Omega}}$. The corresponding 2-vectors $\bar{F}$ and $\bar{G}$ are easily introduced making use of the isomorphism between 2-forms and 2-vectors defined by the volume 4-form $\omega=d x \wedge d y \wedge d z \wedge d \xi$ according to

$$
G=-i_{\bar{F}} \omega, \quad F=i_{\bar{G}} \omega: \rightarrow \quad \bar{\Omega}=\bar{F} \otimes e_{1}+\bar{G} \otimes e_{2} .
$$

We turn now to the corresponding balance law, it reeds:

$$
i \frac{\vee}{\Omega} \mathbf{d} \Omega=0
$$

i.e., the " $\vee$ "-flow of $\overline{\boldsymbol{\Omega}}$ across the change $\mathbf{d} \boldsymbol{\Omega}$ of $\boldsymbol{\Omega}$ does NOT lead to losses. It has to be noted, that this balance law is written down without making use of (pseudo)metric, the volume form $\omega$ serves sufficiently well. We obtain:

$$
\begin{gathered}
i_{\overline{\mathbf{\Omega}}}^{\vee} \mathbf{d} \mathbf{\Omega}=i_{\left(\bar{F} \otimes e_{1}+\bar{G} \otimes e_{2}\right)}^{\vee}\left(\mathbf{d} F \otimes e_{1}+\mathbf{d} \mathbf{G} \otimes e_{2}\right) \\
=i_{\bar{F}} \mathbf{d} F \otimes e_{1} \vee e_{1}+i_{\bar{G}} \mathbf{d} G \otimes e_{2} \vee e_{2}+\left(i_{\bar{F}} \mathbf{d} G+i_{\bar{G}} \mathbf{d} F\right) \otimes e_{1} \vee e_{2}=0 .
\end{gathered}
$$

So, equation (13) gives the following three equations 


$$
i_{\bar{F}} \mathbf{d} F=0, \quad i_{\bar{G}} \mathbf{d} G=0, \quad i_{\bar{F}} \mathbf{d} G+i_{\bar{G}} \mathbf{d} F=0 .
$$

We have here maximum 12 equations for the 12 components of $(F, G)$.

We give now another form to equations (14) making use of the Poincare isomorphisms $\mathfrak{D}$ and $\mathbb{D}$, recalling: $\mathbb{D}_{n-p} \circ \mathfrak{D}^{p}=(-1)^{p(n-p)} i d$ and $\mathfrak{D}^{n-p} \circ \mathbb{D}_{p}=(-1)^{p(n-p)} i d$, also, the divergence operator $\delta^{p}=(-1)^{p} \mathbb{D}_{n-p+1} \circ \mathbf{d} \circ \mathfrak{D}^{p}$. Clearly, $\delta^{p}$ maps $p$-vector fields to $(p-1)$-vector fields. Since in our case

$$
G=-i_{\bar{F}} \omega=-\mathfrak{D} \bar{F}, \quad F=i_{\bar{G}} \omega=\mathfrak{D} \bar{G},
$$

where $\bar{F}$ and $\bar{G}$ are bi-vector fields, then $\delta \bar{F}$ and $\delta \bar{G}$ will be just vector fields. We obtain:

$$
\begin{gathered}
\mathfrak{D}(\bar{F} \wedge \delta \bar{G})=i_{\delta \bar{G}} \mathfrak{D}(\bar{F})=i_{\delta \bar{G}}\left(i_{\bar{F}} \omega\right)=i_{\delta \bar{G}}(-G)=\mathfrak{D}(\delta \bar{G} \wedge \bar{F})=i_{\bar{F}} \mathfrak{D} \delta \bar{G} \\
\quad=i_{\bar{F}} \mathfrak{D}^{1}(-1)^{2} \mathbb{D}_{3} \mathbf{d} \mathfrak{D}^{2} \bar{G}=i_{\bar{F}}(-i d) \mathbf{d} \mathfrak{D}^{2} \bar{G}=-i_{\bar{F}} \mathbf{d}\left(i_{\bar{G}} \omega\right)=-i_{\bar{F}} \mathbf{d} F
\end{gathered}
$$

So, $i_{\delta \bar{G}} G=i_{\bar{F}} \mathbf{d} F$. In the same way following the same line of transformations, from $\mathfrak{D}(\bar{F} \wedge \delta \bar{F})$, $\mathfrak{D}(\bar{G} \wedge \delta \bar{F}), \mathfrak{D}(\bar{G} \wedge \delta \bar{G})$ we obtain consecutively

$$
i_{\delta \bar{F}} F=i_{\bar{G}} \mathbf{d} G, \quad i_{\delta \bar{F}} G=-i_{\bar{F}} \mathbf{d} G, \quad i_{\delta \bar{G}} F=-i_{\bar{G}} \mathbf{d} F .
$$

Hence, equations (14) are equivalent to

$$
i_{\delta \bar{G}} G=0, \quad i_{\delta \bar{F}} F=0, \quad i_{\delta \bar{F}} G+i_{\delta \bar{G}} F=0 .
$$

Note that, from algebraic viewpoint, each of the two equations $i_{\delta \bar{G}} G=0, i_{\delta \bar{F}} F=0$, represents a homogeneous algebraic system of equations for the components of $\delta \bar{G}$ and $\delta \bar{F}$, which components in the both cases are just four. Since the nonlinear solutions would require $\delta \bar{G} \neq 0$ and $\delta \bar{F} \neq 0$, a nonzero nonlinear solution would be possible only if $\operatorname{det}\left\|\left(F_{\mu \nu}\right)\right\|=0$ and $\operatorname{det}\left\|\left(G_{\mu \nu}\right)\right\|=0$, i.e., when $<\alpha, \mathbf{B}>=<\beta, \mathbf{E}>=0$, or, $F \wedge F=0, G \wedge G=0$. In the Minkowski metric case this corresponds to $F_{\mu \nu}(* F)^{\mu \nu}=0$, which means euclidean orthogonality of $\mathbf{E}$ and $\mathbf{B}: \mathbf{E} . \mathbf{B}=0$.

Summing up the above three equations (15) we obtain

$$
i_{(\delta \bar{F}+\delta \bar{G})}(F+G)=i_{\delta(\bar{F}+\bar{G})}(F+G)=0
$$

and since in the nonlinear case $(\delta \bar{F}+\delta \bar{G}) \neq 0$ in general, we must have $\operatorname{det}\left\|(F+G)_{\mu \nu}\right\|=0$, i.e.,

$$
(F+G) \wedge(F+G)=F \wedge F+2 F \wedge G+G \wedge G=2 F \wedge G=2(<\alpha, \mathbf{E}>-<\beta, \mathbf{B}>)=0 .
$$

The two cases $\delta \bar{F}=\delta \bar{G} \neq 0$ and $\delta \bar{F}=-\delta \bar{G} \neq 0$ also require $<\alpha, \mathbf{E}>=<\beta, \mathbf{B}>$. In the Minkowski metric case this is equivalent to $F_{\mu \nu} F^{\mu \nu}=0$, which in the euclidean metric terms means $\mathbf{E}^{2}=\mathbf{B}^{2}$. Hence, in terms of Minkowski spacetime we can say: all nonlinear solutions require null nature of the bi-vector fields $\bar{F}$ and $\bar{G}$, as well as null nature of the 2-forms $F=\mathfrak{D} \bar{G}$ and $G=-\mathfrak{D} \bar{F}$.

Finally, we may say that all nonlinear solutions of (14) require $i \stackrel{\vee}{\vee} \Omega=0$, which leads to $\mathcal{L}_{\bar{\Omega}}^{\vee} \Omega=0$.

The energy-momentum tensor is the same as in the linear relativistic case (8). Here we approach the conservation aspects as follows. It should be clear that the object considered propagates, so, every local conservation aspect must be considered with respect to the vector field $X$, determining the corresponding propagation. Therefore, the energy tensor (8) must be appropriately projected along this vector field $X$, and the quantity under consideration should look like $\mathbf{V}=X^{\sigma} \mathbb{T}_{\sigma}^{\mu} \frac{\partial}{\partial x^{\mu}}$. Since this vector field $\mathbf{V}$ must NOT lose, or gain, anything to/from the volume where it propagates, 
the coordinate free adequate image to this property will require the corresponding Lie derivative of the 4 -volume form $\omega$ with respect $\mathbf{V}$ to be zero: $L_{\mathbf{V}} \omega=0$. Now, since $\mathbf{d} \omega=0$, for the Lie derivative we obtain

$$
0=L_{\mathbf{V}} \omega=\mathbf{d} i_{\mathbf{V}} \omega+i_{\mathbf{V}} \mathbf{d} \omega=\mathbf{d} i_{\mathbf{V}} \omega=\mathbf{d} \mathfrak{D}(\mathbf{V})
$$

The obtained 3-form $\mathfrak{D}(\mathbf{V})$ is closed, so the corresponding to $X$ conserved quantity is explicitly represented, and restricting this 3 -form to the 3 -space, we can integrate over the occupied by the object 3 -volume in order to compute the integral value of this quantity, carried by the electromagnetic object considered.

It deserves now noting the following. In this coordinate free pre-metric formulation we may forbid about how we came to the components of $F$ and $G$, i.e., each of the new constituents $F$ and $G$ has six components and that's all, getting back to $(\mathbf{E}, \mathbf{B} ; \alpha, \beta)$ is not necessary.

The equations obtained suggest some connection with the concepts of absolute and relative integral invariants of a vector field $X$ on a manifold $M$ introduced and used by E.Cartan [23]: these are differential forms $\alpha \in \Lambda(M)$ satisfying respectively the relations $i(X) \alpha=0, i(X) \mathbf{d} \alpha=0$, leading to $L_{X} \alpha=0$, and just $i(X) \mathbf{d} \alpha=0$. Our relations may be considered as corresponding extensions:

- a vector field $\rightarrow$ vector valued multivector field

- a differential form $\rightarrow$ vector valued differential form,

and these extensions allow to make use of the mentioned in Sec.2 extension of the Lie derivative of a differential form along multivector fields.

The new moment in our extension is that we consider vector valued multivectors along which vector valued forms to be differentiated with respect to some bilinear map $\varphi: V \times V \rightarrow W$, where $W$ is appropriately determined vector space. In our case $\varphi=\mathrm{V}$, which corresponds to the specific kind of interaction between the two subsystems: absence of non-zero interaction stress-energy.

In general, we note that, the triple $(V, W ; \varphi)$ determines possible interactions among the subsystems of the field object considered. In our considerations these subsystems are formally represented by the vector components of the multi-vector $\bar{\Omega}$ and the vector components of the multi-differential form $\boldsymbol{\Omega}$, i.e., $(\bar{F}, F)$ and $(\bar{G}, G)$.

\section{Conclusion}

From general point of view, getting knowledge for the internal compatibility and external stability of a physical object is being done by measuring the corresponding to these physical appearances appropriate physical quantities. Such physical quantities may vary in admissible, or not admissible extent: in the first case we talk about admissible changes, and in the second case we talk about changes leading to destruction of the object. Formally, this is usually verified by calculating the flow of the formal image of the (sub)system considered through its appropriately modelled change, as it is seen, e.g., in (11),(12),(13), i.e., by means of finding corresponding differential self flows of the subsystems, e.g., $i_{\bar{F}} \mathbf{d} F$, and differential mutual flows among the subsystems, e.g., $i_{\bar{F}} \mathbf{d} G$. Since every measuring process requires stress-energy-momentum transferring between the object studied and the measuring system, the role of finding corresponding tensor representatives of these change-objects and of the corresponding flows is of serious importance. Therefore, having adequate stress-energy-momentum for the considered case, the clearly individualized tensor members of its divergence represent qualitatively and quantitatively important aspects of the intrinsic dynamical 
nature of the object considered. This view motivated the above given approach to find appropriate description of electromagnetic field objects.

The existing knowledge about the structure and internal dynamics of free electromagnetic field objects made us assume the notion for two partner-fields internal structure, formally represented by $(F, \bar{F} ; G, \bar{G})$ on $\mathbb{R}^{4}$. Each of these two partner-fields is built of two formal constituents, and each partner-field is able to carry local stress-energy-momentum, allowing internal local "intercommunication" between its constituents during the local interaction with its partner-field. The two subsystems carry equal local energy-momentum densities, and realize local mutual energy exchange without available interaction energy. Moreover, they strictly respect each other: the exchange is simultaneous and in equal quantities, so, each of the two partner-fields keeps its identity and recognizability. The corresponding internal dynamical structure appropriately unifies translation and rotation through unique space-time propagations as a whole with the fundamental velocity. All linear solutions to (14) represent Maxwell charge-free solutions. As it was shown [21], in the corresponding Minkowski space-time consideration the new nonlinear solutions, i.e., those satisfying $\mathbf{d} F \neq 0, \mathbf{d} * F \neq 0$, are time-stable, they admit FINITE SPATIAL SUPPORT, and demonstrate compatible translational-rotational dynamical structure.

\section{Appendix}

A natural formal extension of the final formulation of our nonlinearization is the following.

Consider an oriented $4 n$-manifold $\mathcal{M}=\left(M^{4 n}, \omega\right)$. Denote by $\Lambda^{2 n}(\mathcal{M})$ the $2 n$-differential forms on $\mathcal{M}$, and by $\mathfrak{X}^{2 n}(\mathcal{M})$ the the antisymmetric $2 n$-vector fields on $\mathcal{M}$. Let now $\left(\bar{F}_{1}, \bar{F}_{2}, \ldots, \bar{F}_{n}\right)$ be a set of lineary independent $2 n$-vector fields and $\left(\bar{G}_{1}, \bar{G}_{2}, \ldots \bar{G}_{n}\right)$ be another set of lineary independent $2 n$-vector fields, i.e., members of $\mathfrak{X}^{2 n}(\mathcal{M})$. Let $(i=1,2, \ldots, n)$ and $(j=n+1, n+2, \ldots, 2 n)$. The Poincare isomorphysms generate the corresponding $2 n$-differential forms $\left(F^{i}, G^{j}\right)$ :

$$
F^{i}=i_{\bar{G}_{j}} \omega, \quad G^{j}=-i_{\bar{F}_{i}} \omega, \quad j=n+i .
$$

Let $\mathbf{V}$ be a $2 n$-dimensional real vector space with basis $\left(e_{1}, e_{2}, \ldots, e_{2 n}\right)$, and $\vee: \mathbf{V} \times \mathbf{V} \rightarrow(\mathbf{V} \vee \mathbf{V})$ denote the symmetrized tensor product. We can now define the $\mathbf{V}$-valued objects (summation along $i$ and $j$ )

$$
\Omega=F^{i} \otimes e_{i}+G^{j} \otimes e_{j}, \quad \bar{\Omega}=\bar{F}^{i} \otimes e_{i}+\bar{G}^{j} \otimes e_{j},
$$

and the $\mathbf{V} \vee \mathbf{V}$-valued self-mutual flow

$$
i_{\bar{\Omega}}^{\vee} \mathbf{d} \Omega=i_{\bar{F}_{i}} \mathbf{d} F^{i} \otimes\left(e_{i} \vee e_{i}\right)+i_{\bar{G}_{j}} \mathbf{d} G^{j} \otimes\left(e_{j} \vee e_{j}\right)+\left(i_{\bar{F}_{i}} \mathbf{d} G^{j}+i_{\bar{G}_{j}} \mathbf{d} F^{i}\right) \otimes\left(e_{i} \vee e_{j}\right)
$$

So, if this self-mutual flow is zero : $i_{\bar{\Omega}}^{\vee} \mathbf{d} \Omega=0$, we obtain the equations

$$
i_{\bar{F}_{i}} \mathbf{d} F^{i}=0, \quad i_{\bar{G}_{j}} \mathbf{d} G^{j}=0, \quad i_{\bar{F}_{i}} \mathbf{d} G^{j}+i_{\bar{G}_{j}} \mathbf{d} F^{i}=0,
$$

meaning that every sub-object $\left(F^{i}, \bar{F}_{i}\right)$ stays recognizable during its interaction with all of $\left(G^{j}, \bar{G}_{j}\right)$, and every sub-object $\left(G^{j}, \bar{G}_{j}\right)$ stays recognizable during its interaction with all of $\left(F^{i}, \bar{F}_{i}\right)$.

Finally we mention that if the sub-objects are able to choose interaction partners inside this system, the corresponding partnership can be specialized by replacing the symmetrized tensor product " $\vee$ " with another appropriate bilinear map $\varphi: \mathbf{V} \times \mathbf{V} \rightarrow \mathbf{W}$ to some vector space $\mathbf{W}$. 


\section{References}

[1]. W.H. Greub,Multilinear Algebra, Springer Verlag, second edition, 1978, New York; W.H. Greub, S.Halperin, R.Vanstone, Connections, Curvature, and Cohomology, Vols. I-II, Academic Press, 1972-1973

[2]. W.M. Tulczyjew, The Graded Lie Algebra of Multivector Fields and the Generalized Lie Derivative of Forms, Bull. Acad. Pol. Sci. SMAP 22 (1974) 937-942; The Poisson Bracket for Poisson Forms in Multisymplectic Field Theory, arXiv: math-ph/0202043v1

[3]. D.A.Delphenich, http://www.neo-classical-physics.info/electromagnetism-and- wave-theory.html, "Pre-metric Electromagnetism", parts I, II, e-book.

[4]. M. Born, L.Infeld, Proc.Roy.Soc., A 144, 425 (1934)

[5]. W. Heisenberg, H. Euler, Zeit.Phys., 98, 714 (1936)

[6]. M. Born, Ann. Inst. Henri Poincare, 7, 155-265 (1937).

[7]. J. Schwinger, Phys.Rev. ,82, 664 (1951).

[8]. H. Schiff, Proc.Roy.Soc. A 269, 277 (1962).

[9]. J. Plebanski, Lectures on Nonlinear Electrodynamics, NORDITA, Copenhagen, 1970.

[10]. G. Boillat, Nonlinear Electrodynamics: Lagrangians and Equations of Motion, J.Math.Phys. 11, 941 (1970).

[11]. B. Lehnert, S. Roy, Extended Electromagnetic Theory, World Scientific, 1998.

[12]. D.A. Delphenich, Nonlinear Electrodynamics and $Q E D$, arXiv:hep-th/0309108, (good review article).

[13]. B. Lehnert, A Revised Electromagnetic Theory with Fundamental Applications, Swedish Physic Arhive, 2008.

[14]. D. Funaro, Electromagnetsm and the Structure of Matter, Worldscientific, 2008; also: From photons to atoms, arXiv: gen-ph/1206.3110 (2012).

[15]. E. Schrodinger, Contribution to Born's new theory of electromagnetic feld, Proc. Roy. Soc. Lond. A 150, 465 (1935).

[16]. G. Gibbons, D. Rasheed, Electric-magnetic duality rotations in non-linear electrodynamics, Nucl. Phys. B 454185 (1995) hep-th/9506035.

[17] R. Kerner, A.L. Barbosa, D.V. Gal'tsov, Topics in Born-Infeld Electrodynamics, arXiv: hep-th/0108026 v2

[18]. S.G.Donev, A particular nonlinear generalization of Maxwell equations admitting spatially localized wave solutions, Compt.Rend.Bulg.Acad.Sci., vol.34, No.4 (1986).

[19]. S. Donev, M. Tashkova, Energy-momentum directed nonlinearization of Maxwell's pure field equations, Proc.R.Soc.Lond. A , 1993, 443, 301-312.

[20]. S. Donev, M. Tashkova, Energy-Momentum Directed Nonlinearization of Maxwell's Equations in the Case of a Continuous Medium /Donev, S., Tashkova, M./, Proc.R.Soc. Lond.A 450, 281 (1995)

[21]. S. Donev, M. Tashkova, Geometric View on Photon-like Objects, LAMBERT Academic Publishing, 2014 (also: arXiv,math-ph, 1210.8323v2)

[22]. S. Donev, Geodesic Vector Fields on Minkowski Space-Time and (3+1)-Solitary Waves, Commun.JINR - Dubna, E2-88-107.

[23]. E. Cartan, Lecons sur les invariants integraux. Cours professe a la Faculte des sciences de Paris, 1920-1921. 